The subjoined observations were made from approximately 500 cases seen in private practice. As elsewhere, the fatalities were chiefly among the 20 to 35 age group. Similarly the prognosis was better for those over 40 . Elderly patients were rare. It was a question whether it was more frequent for a child or an adult to bring the disease into the family.

Epistaxis was common, occurring in from 10 to 15 per cent. of the cases. Some of these resulted in pneumonia. The headache and backache frequently persisted for three or four days. The temperature on the first day was between 101 and 104. A feature of the temperature was its variability during the day, rising and falling at irregular intervals. In the ordinary case the temperature persisted for from two to five days. Sweating was common even without medication or hot drinks. The bowels were inclined to be constipated. There was a general but slight enlargement of the lymph nodes. The spleen was not palpable. The pulse was relatively slow in many "cases, but was not always so.

In regard to treatment, no particular drug had special virtues. Rest in bed, fresh air, light, nourishing food, plenty of water and open bowels were the main reliances. A simple expectorant cough mixture, sometimes with the addition of codein, sufficed for internal medication in the ordinary case. Alcohol rubs were soothing when the temperature was high. For the heaviness and soreness in the chest, home-made mustard plasters undoubtedly gave relief. The "fever and pain" powders or capsules, as were ordinarily prescribed, frequently only increased the prostration, and in a few cases in which the patient had taken a number of them, it seemed to prolong their convalescence. This type of medication was frequently more harmful for the patient than the pain for which it was intended.

In the pneumonias, stimulation did not seem to stimulate. It was rare to find pneumonia develop in a patient who took to bed at the onset of his infiuenza, and have it set in before convalescence, but it did occur. Pneumonia usually occurred after too early getting out of bed, or from too strenuous efforts after or during convalescence.

Three cases of the meningeal type of influenza were encountered. Prompt spinal puncture with withdrawal of excess fluid which was under pressure gave immediate relief. These cases were recognized by unconsciousness chiefly, with variable rigidity of neck, convulsions, involuntaries, dilatation of pupils and vomiting. In one of these cases, in which spinal puncture was delayed, recovery was slower than in the other two.

Harry Berman, M.D., Hartford, Conn.

\section{PREVENTION OF INFLUENZA BY OSMOTIC ACTION IN AIR PASSAGES}

To the Editor:-The infection of influenza unquestionably enters the system through the nasal spaces and upper air passages. This fact is practically proved by a mode of disinfection and cleansing of those spaces and surfaces which I have suggested and used in the case of many thousand individuals who would ordinarily be considered likely subjects to infection. Infection was prevented in practically 100 per cent. when the treatment was properly used.

The tortuosities of the nasal and postnasal spaces and their great extent make the use of the many remedies suggested ineffective, because, however excellent they may be in themselves, they do not reach all the surfaces involved and they furthermore lack certain physical qualities necessary to invite a sufficient and strong osmotic action.

A powder composed of impalpable boric acid, 95 parts, sodium bicarbonate, 3 parts, and calomel, 2 parts, thoroughly triturated, is the basis of treatment.

With a good powder blower, this powder is freely blown into the nose, and will reach every recess in the upper air .passages, and does not seem irritating to any extent. This powder, by abstraction of liquids from the moist surfaces, dissolves, excepting the calomel, and will shortly cause a mucoserous flow induced by osmotic pressure, and during this process the membranes involved are thoroughly washed and disinfected by virtue of the double osmotic current induced. This mucoid secretion can then be discharged, and the process repeated after from four to six hours. Ordinarily, the use of this powder once a day will suffice to keep the nasal membranes in fine condition and free from infections.

James A. BaCh, M.D., Milwaukee.

\section{THE CIVILIAN PHYSICIAN'S SACRIFICE}

To the Editor:-May I ask why as my husband was a victim on the long list of doctors who succumbed to the terrible epidemic last month no mention is made in any periodical or public newspaper of these noble fellows who gave their lives in fighting this disease? Are they not to be mentioned in any way as heroes?

The sacrifices of physicians in this section of our country was greater than the people of closely populated districts during the past four years of war can conceive, as the distance they must cover is great, and doctors are few. My husband for the past year has practiced alone in this county his nearest town being 28 miles away, the farthest, 75 miles. He could not enter the Army on account of being tuberculous. When he died he had worked night and day for two weeks alone trying to save people in this county.

Another tuberculous doctor from an adjacent county came over and took up the fight, leaving a little doctor in his place who left a tuberculosis sanatorium in El Paso, to aid the people. I am sure similar cases could be brought to mind over our country. Is there no memorial for these gallant men?

Mrs. Longino, Fort Stockton, Texas.

\section{THREE SIGNS OF SOME VALUE IN THE DIAGNOSIS OF UNUSUAL CASES OF INFLUENZA}

To the Editor:-The last epidemic of influenza has been confined almost entirely to inflammatory changes in the respiratory tract. While it is true that there have been occasional instances in which the whole brunt of the disease has been borne by other systems, these could be diagnosticated only by inference as (1) the presence of the epidemic; (2) attacks of well-marked respiratory influenza in other members of the same family, or (3) previous similar attacks, unquestionably of infuenzal nature in the same individual. Even here, in many instances, on close questioning, it could be ascertained that there was an occasional sneeze four or five days before the onset of the disease.

In these out-of-the-ordinary attacks, the nervous system was the one commonly involved. However, 95 per cent. of the cases seen in this epidemic involved the respiratory system, and the diagnosis was almost always made within two or three minutes after seeing the patient. Now and again in an isolated case of this type the question was raised in the mind of the practitioner "whether or not one was dealing with an influenza or with an ordinary head cold or coryza," such as is not uncommon at this time of the year. The absence of early rise of temperature and pulse and respiration changes in some of the influenza cases made this often a puzzling problem. Most physicians "played safe" and diagnosticated all of such cases as influenza. This was eminently fit and proper.

1. In a large number of true cases of influenza of the respiratory tract I have noticed a sign that may at times be of some help from a diagnostic standpoint. It has not to my knowledge been described before. It consists of a very marked redness of the opening of the salivary or Steno's duct, just opposite the second molar tooth. The punctum is usually swollen and raised above the level of the cheek, and the red spot is seen in the center. Mere swelling of the punctum has no diagnostic import. It may easily be inspected by placing a spoon handle in the side of the mouth and retracting it. If the sign is present on one side it is almost always visible on the other as well. It is not invariably present, but can be seen in at least 70 per cent. of the cases. 